University of Wollongong

Research Online

Faculty of Engineering and Information

Faculty of Engineering and Information

Sciences - Papers: Part B

Sciences

2020

Gait asymmetry and variability in older adults during long-distance walking: Implications for gait instability

Duo Wong

Wing Lam

Winson Lee

University of Wollongong, ccwlee@uow.edu.au

Follow this and additional works at: https://ro.uow.edu.au/eispapers1

Part of the Engineering Commons, and the Science and Technology Studies Commons

Research Online is the open access institutional repository for the University of Wollongong. For further information contact the UOW Library: research-pubs@uow.edu.au 


\title{
Gait asymmetry and variability in older adults during long-distance walking: Implications for gait instability
}

\author{
Abstract \\ 2019 Background: Physical exercise, such as walking, is imperative to older adults. However, long- \\ distance walking may increase walking instability which exposes them to some fall risks. Objective: To \\ evaluate the influence of long-distance walking on gait asymmetry and variability of older adults. Method: \\ Sixteen physically active older adults were instructed to walk on a treadmill for a total of $60 \mathrm{~min}$. Gait \\ experiments were conducted over-ground at the baseline (before treadmill-walk), after first $30 \mathrm{~min}$ \\ (30-min) and second $30 \mathrm{~min}(60-\mathrm{min})$ of the walk. In addition to spatiotemporal parameters, median \\ absolute deviation of the joint angular velocity was measured to evaluate gait asymmetry and gait \\ variability. Findings: There were significant differences in the overall asymmetry index among the three \\ time instances (Partial $\eta 2=0.77, p<.05$ ), predominantly contributed by the ankle (Partial $\eta 2=0.31, p<$ \\ .017). Long-distance walking significantly increased the average and maximum median absolute \\ deviation of the ankle at both sides $(W \geq 0.19, p<.05)$, and knee at the non-dominant side $(W=0.44, p<$ \\ .05). Interpretation. At 30-min, the older adults demonstrated a significantly higher asymmetry and \\ variability at the ankle, which implied higher instability. Continue walking for an additional $30 \mathrm{~min}$ (60-min) \\ further increased variability of the non-dominant limb at the knee joint. Walking for 30 min or more could \\ significantly reduce walking stability.

\section{Disciplines} \\ Engineering | Science and Technology Studies

\section{Publication Details} \\ Wong, D., Lam, W. \& Lee, C. (2020). Gait asymmetry and variability in older adults during long-distance \\ walking: Implications for gait instability. Clinical Biomechanics, 72 37-43.
}


1 Gait Asymmetry and Variability in Older Adults during Long-distance

2 Walking: Implications for Gait Instability

3 Clin Biomech (Bristol, Avon). 2019;72:37-43

4

5 Duo Wai-Chi Wong ${ }^{1}$, Wing-Kai Lam²,3,4, Winson Chiu-Chun Lee ${ }^{5, *}$

7 'Department of Biomedical Engineering, Faculty of Engineering, The Hong Kong

8 Polytechnic University, Hong Kong, China

$9 \quad{ }^{2}$ Department of Kinesiology, Shenyang Sport University, Shenyang, China

$10{ }^{3}$ Guangdong Provincial Engineering Technology Research Center for Sports

11 Assistive Devices, Guangzhou Sport University, Guangzhou, China

$12{ }^{4}$ Li Ning Sports Science Research Center, Li Ning (China) Sports Goods Company,

13 Beijing, China

$14{ }^{5}$ School of Mechanical, Materials, Mechatronic and Biomedical Engineering,

15 University of Wollongong, New South Wales, Australia

17 Correspondence: Dr. Winson Chiu-Chun Lee

18 School of Mechanical, Materials, Mechatronic and Biomedical Engineering,

19 University of Wollongong, New South Wales, Australia

20 Email: Winson_lee@uow.edu.au

21

22 World Count: Main-text: 2739, Abstract: 234

23 


\section{Abstract}

25 Background: Physical exercise, such as walking, is imperative to older adults.

26 However, long-distance walking may also increase walking instability which

27 exposes them to some fall risks.

28 Objective: To evaluate the influence of long-distance walking on gait asymmetry 29 and variability of older adults.

30 Method: Sixteen physically active older adults were instructed to walk on a

31 treadmill for a total of 60 minutes. Gait experiments were conducted over-ground

32 at the baseline (before treadmill-walk), after first 30-minute (30-min) and second

33 30-minute (60-min) of the walk. In addition to spatiotemporal parameters, median

34 absolute deviation of the joint angular velocity were measured to evaluate gait

35 asymmetry and gait variability.

36 Findings: There were significant differences in the overall asymmetry index among

37 the three time instances (Partial $\eta^{2}=0.77, p<0.05$ ), predominantly contributed by

38 the ankle (Partial $\left.\eta^{2}=0.31, p<0.017\right)$. Long-distance walking significantly altered

39 the average and maximum median absolute deviation of the ankle at both sides

$40 \quad(\mathrm{~W} \geq 0.19, p<0.05)$, and knee at the non-dominant side $(\mathrm{W}=0.44, p<0.05)$.

41 Interpretation: At 30-min, the older adults demonstrated a significantly higher 42 asymmetry and variability at the ankle, which implied higher instability. Continue

43 walking for an additional 30 minutes (60-min) further increased variability of the 
44 non-dominant limb at the knee joint. Walking for 30 minutes or more could induce

45 significant reduction in walking stability.

46

47 Keywords: Prolonged walking; prolonged exercises; fatigue; kinematics;

48 


\section{1. Introduction}

50 The advantages of physical exercises in older adults are well recognized (Lim and

51 Taylor, 2005). However, more than half of the older adults reported that they did

52 not have sufficient physical exercises (Lim and Taylor, 2005). Walking is regarded

53 as one of the most accessible types of exercises for older adults, which could occur

54 through activities of daily living without any equipment (Chastin et al., 2019). Older

55 adults are recommended to walk 10,000 steps per day for improved health

56 (Duncan et al., 2014). However, a majority of healthy older adults can only walk

573,000 step a day which barely reach the target (Tudor-Locke and Bassett Jr, 2004).

58 Blindly pushing older adults to achieve a generalized walking goal may do more

59 harm than good. Older adults gradually decrease their muscle strength by $1.5 \%$

60 and $3.0 \%$ yearly after the age of 50 and 60 , respectively (Van Kan, 2009). Muscle

61 weakening and fatigue reduce walking stability, inducing higher chance of fall to

62 older adults when they try to achieve some walking goals (Helbostad et al., 2010)

66 While some studies have successfully linked responses to perturbation to risk of

67 falling (Bohm et al., 2015), some other studies have used gait variability which is

68 often measured in laboratory settings to evaluate walking stability and predict risk

69 of falling as reviewed in Hamacher et al., 2011. High inter-limb asymmetry and gait

70 variability could reflect some impaired motor control leading to errors in foot 
71 placement (Maki, 1997). Stride-to-stride and step-to-step variability of discrete gait

72 variables, including step width and step stance, were found to be correlated with

73 falls risk among active community dwelling older adults (Paterson et al., 2011).

74 Variability of body sway has also been identified as a predictor of fall and

75 imbalance (Greene et al., 2012; Jansen et al., 2014). Meanwhile, some studies

76 focused on a continuous analysis of the variability of knee and hip joint angles and

77 angular velocities throughout the stance phase, and identified the relationships

78 among functional impairment, muscle fatigue, and poor coordination (Cortes et al.,

79 2014; Fallah-Yakhdani et al., 2012; Smith et al., 2014). Increased gait asymmetry

80 generally reflects poor coordination that disturbs gait stability increasing falls risk

81 (Hausdorff, 2007).

82

83 There is abundant of research studying the gait stability of older adults as reviewed

84 in Hamacher et al., 2011. For example, the comparison between younger and older

85 adults, as well as fallers and non-fallers, provided some understandings on the

86 decline of walking functions and instability upon aging (Beauchet et al., 2009).

87 However, there is limited research on the study of the changes in gait stability

88 during prolonged physical exercises, such as long-distance walking. Such

89 research is warranted as it allows better understanding of the walking limit of older

90 adults. It may also aid in better design of physical training and rehabilitation regime

91 given that physical training can improve motor control and improve gait stability

92 (Donath et al., 2016). 
93 The main objective of this study is to investigate the effects of long-distance

94 walking on variability and inter-limb symmetry of joint angular velocities. We

95 hypothesize that gait variability and inter-limb asymmetry increase after some

96 instances of long-distance walking. This study would shed some light on the

97 physical limit of the older adults in walking.

\section{2. Methods}

992.1 Participants

100 Twenty older adults aged over 65 were recruited in this study with convenient 101 sampling. There were four participants dropped out from the study. Three of them

102 stopped the experiment due to physical exhaustion. Another participant was 103 unable to arrange another required gait test. The sample size of 16 produced 104 statistical power of 0.75 , assuming medium effect size and a level of significance 105 of $5 \%$. The participants were independent to walk without use of walking aids. 106 Exclusion criteria included cardiovascular, pulmonary diseases, cancer, 107 uncontrolled hypertension, diabetes, lower-limb pain or deformities. Participants 108 were excluded if they had a history of fall in the past 12 months. The 16 participants 109 (11 males, 5 females) had a mean age of 70 (SD: 5.0). Their mean height and 110 mass were $163.5 \mathrm{~cm}$ (SD: 7.3$)$ and $63.3 \mathrm{~kg}$ (SD: 9.2), respectively.

111 This experiment was approved by the institutional review board of the university

112 (Ref. No.: HSEARS20151016007). All participants signed an informed consent

113 statement after receiving the oral and written descriptions of the experimental 114 procedure prior to the start of the experiment. 


\section{2.2 Equipment and Procedure}

116 The participants were asked to walk on a treadmill at their self-selected

117 comfortable speed for two consecutive 30-minute walking sessions. Gait

118 assessments were conducted at three time conditions: prior to the walking session

119 (baseline), after the first 30-minute walking session (30-min), and the second 30-

120 minute walking session (60-min). Before the gait assessments, the participants

121 were given a 2-minute familiarization time to adapt environment changes from

122 treadmill to over-ground walking.

123 Gait assessments were conducted over-ground in a 10-m gait laboratory equipped

124 with the motion capture system (Vicon, Oxford Metrics Ltd., Oxford, UK) and force

125 plates, which was sampled at $200 \mathrm{~Hz}$ and $1000 \mathrm{~Hz}$, respectively. Twenty-four

126 infrared reflective markers were placed over both limbs at the anterior and superior

127 iliac spines and crests, greater trochanters, middle of thighs and shanks anteriorly,

128 medial and lateral femoral condyles and malleoli, foot dorsum, and heels (Winter, 129 1991).

130 A trial with both left and right clean footfalls on separate force plates, which were 131 located at the center of the runway, was regarded as a successful trial. The ground 132 reaction force data were used to define the start and end of the gait cycle for 133 subsequent analyses. Five successful trials were collected and only the second to 134 the fourth trials were extracted to minimize irregularities due to gait initialization 135 and termination (Wong et al., 2015). The angular velocities of the ankle, knee and 136 hip joints at the dominant and non-dominant limbs in the sagittal plane were 
137 extracted from the Vicon Nexus Software and subsequently processed in Visual

138 3D (C-Motion Inc., USA) using a $4^{\text {th }}$ order Butterworth low-pass filter with cutoff

139 frequency of $6 \mathrm{~Hz}$. Spatiotemporal parameters, including walking speed, step

140 length and stance time of the dominant and non-dominant sides were also

141 extracted. The dominant limb was determined by asking the participants the side

142 of the limb they would use to kick a ball (Chapman et al., 1987).

\section{2.3 Data Management}

144 The time-series of the data were normalized to 101 time-points that represented 0

145 to $100 \%$ gait cycle (Bruijn et al., 2013). Symmetry index (SI), which was calculated

146 by the percentage ratio of the data difference to the data mean, was used to

147 quantify the symmetry between dominant and non-dominant limbs. The average

148 SI was calculated by averaging all Sls across the time-series.

149 Median Absolute Deviation (MAD) is a commonly used variability index which is

150 defined as the median of the deviations from the data median and was calculated

151 using the three successful trials for each of the time conditions (baseline, 30-min,

152 and 60-min) (Bruijn et al., 2013). The maximum MAD and mean MAD (i.e., 153 averaging all MADs across the time-series) were extracted for analysis (Wong et 154 al., 2015).

\section{$155 \quad 2.4$ Statistical Analysis}

156 MAD and spatiotemporal parameters, including walking speed, step length and

157 stance time of the dominant and non-dominant sides were examined using the 158 non-parametric Friedman test to compare among the baseline, 30-min, and 60- 
159 min conditions since some parameters violated the assumption of normality.

160 Significance level $(\alpha)$ was set at $p=0.05$. Post-hoc analyses with Wilcoxon signed-

161 rank tests were conducted with a Bonferroni correction at $p<0.017$.

162 Regarding SI, a one-way multivariate analysis of variance (MANOVA) with

163 repeated measures was conducted to determine the effect of walking time

164 (baseline, 30-min, and 60-min) on the Sls of the ankle, knee, and hip joints. The

165 outcome of the MANOVA essentially combines these dependent variables in the

166 analysis such that we named it as the overall SI. Significance level $(\alpha)$ was set at

$167 p=0.05$. Univariate and post-hoc analysis with Bonferroni correction would be 168 subsequently conducted when significance was revealed.

170 3. Results

1713.1 Spatiotemporal Parameters

172 As shown in Table 1, there were no significant differences in walking speed, step

173 length of both limbs and stance time of the non-dominant side among the three

174 time conditions. The stance time of the dominant side significantly decreased with

175 time $\left(\chi^{2}(2)=19.27, p<0.05\right)$. Post-hoc test showed that all pairwise comparisons

176 demonstrated statistical differences (baseline vs $30-\min : Z=-3.44 ; 30-\mathrm{min}$ vs $60-$

$177 \min : Z=-2.59$; baseline vs $60-\min : Z=-3.46, p<0.017)$.

$178 \quad 3.2$ Symmetry Index 
179 Pre-hoc test using the Shapiro-Wilk test revealed that all the data were normally

180 distributed $(p>0.05)$; and Pre-hoc test using Pearson correlation revealed that

181 there was no multi-collinearity $(0.2>r>0.6)$; there were linear relationships among

182 the asymmetry data of the three joints, as observed on the scatterplots.

183 MANOVA indicated that the significant difference among the time conditions in the

184 overall SI, $F(6,10)=5.47, p<0.05$; Wilks' $\Lambda=0.23$; partial $\eta^{2}=0.77$ (Table 2).

185 Follow-up univariate test showed that statistically significance only appeared at the

186 ankle angular velocity, $F(1.31,19.61)=6.58, p<0.017$; partial $\left.\eta^{2}=0.31\right)$. Post-

187 hoc analysis revealed that the SI of the ankle was significantly increased after the

188 first 30-minute walking session, $11.88, p<0.017$ (95\% Cl, 4.87 to 18.89), as shown

189 in Figure 1.

\section{3.3 Maximum and Average MAD}

191 In Figure 2, there were statistically significant differences in the average MAD of

192 the ankle joint at both non-dominant $\left(\chi^{2}(2)=6.13, \mathrm{~W}=0.19, p<0.05\right)$ and

193 dominant limbs $\left(\chi^{2}(2)=10.13, \mathrm{~W}=0.32, p<0.05\right)$, and the knee joint at the non-

194 dominant $\operatorname{limb}\left(\chi^{2}(2)=14.00, \mathrm{~W}=0.44, p<0.05\right)$ among the three time conditions.

195 Post-hoc analysis on the ankle joint at the non-dominant limb showed no 196 significance among the pairwise comparisons, despite that significance was 197 demonstrated $\left(\chi^{2}(2)=6.125, p=0.047\right)$. For the ankle joint of the dominant limb, 198 there was a statistically significant increase in 30-min compared with the baseline $199(Z=-3.52, r=0.88, p<0.001)$. Similarly, there were a significant increase in the 
200 non-dominant sided knee joint after the first 30-minute walking $\operatorname{session}(Z=-3.52$, $201 \quad r=0.88, p<0.001)$.

202 In Figure 3, there were statistically significant differences in the maximum MAD of 203 the ankle joint at both non-dominant $\left(\chi^{2}(2)=8.38, W=0.26, p<0.05\right)$ and 204 dominant limbs $\left(\chi^{2}(2)=6.50, W=0.20, p<0.05\right)$, the knee joint at the non205 dominant $\operatorname{limb}\left(\chi^{2}(2)=11.38, \mathrm{~W}=0.36, p<0.05\right)$, and the hip joint at the dominant $206 \operatorname{limb}\left(\chi^{2}(2)=9.13, p<0.05\right)$. Despite overall significance determined in the ankle 207 and hip joints, there were no significance differences among the time points for 208 both limbs in the post-hoc comparison tests. On the other hand, the knee joint at 209 the non-dominant side showed significant increases at 30-min $(Z=-2.48, r=0.62$, $210 p=0.013)$ and $60-\min (Z=-2.59, r=0.65, p=0.010)$, when compared to the 211 baseline condition.

\section{4. Discussion}

213 The present study showed that after 30 minutes of walking the participants 214 significantly increased 1) asymmetry between limbs at the ankle joint, 2) variability 215 of the ankle joint at the dominant limb, and 3) variability of the knee joint at the 216 non-dominant limb. The increased variability at the ankle joint aligned with the 217 findings of previous studies, which indicated that plantarflexors of the dominant 218 limb were more vulnerable to fatigue (Yeung et al., 2012) and that long-distance 219 walking significantly reduced dominant-sided ankle power during push-off phase 220 of the gait (Elhadi et al., 2016). This study also found that stance time of the 221 dominant side was significantly reduced after both 30 and 60 minutes of walking, 
222 which could be induced by the altered kinematics of the ankle. Meanwhile, the 223 significantly higher variability of the non-dominant sided knee joint after 30 minutes 224 of walk could be explained by a previous study (Elhadi et al., 2016), which showed 225 that the knee joint at the non-dominant side appeared to compensate for the 226 fatigued plantarflexors at the dominant side.

227 Although statistically insignificant, there was a trend that while the symmetry of the 228 ankle and hip joints reduced after 30 minutes, they slightly improved after 60 229 minutes. This aligned with a previous study which suggested that the asymmetry 230 and variability improved with further physical exercises and attributed this to the 231 ability of using a compensatory walking mechanism attempting to restore postural 232 balance (Wong et al., 2015). However, there are questions regarding when exactly 233 and how this compensatory walking mechanism is triggered by older adults. Our 234 finding was contrary to the study conducted by Hamacher et al.(2016) which 235 suggested that based on self-reported submaximal exhaustion levels, physical 236 exhaustion increased dynamic stability in young people only but not in older 237 adults. . More investigations regarding the relationship between age and the 238 compensatory mechanism are needed.

239 Both within-subject and between-subject variations, as demonstrated by the MADs 240 and data outliers, were not small in our study. Walking stability depends on 241 individual fitness, musculoskeletal conditions, cognition, neural control and 242 reflex(Krasovsky et al., 2012). In the present study, all our participants were able 243 to walk independently without any diseases which may affect walking. However, 244 the variations among participants still existed as some participants had a less 
245 stable gait in the baseline. Three participants were dropped out in the study due to 246 exhaustion. This could be one limitation of this study as this study potentially 247 excluded some potential participants which might be at risk of falling. While a 248 universal method to classify the frailty of elderly was lacking (Paw et al., 2001), a 249 larger population study enabling the categorization of subject groups according to 250 their level of baseline performance of physical or functional activities is warranted. 251 The association between the baseline performance and intrinsic factors, such as 252 physical fitness and cognitive level, should also be addressed.

253 The long-distance walking was mostly facilitated by a treadmill as it allows for 254 indoor monitoring and cross-study comparison. It should be noted that treadmill 255 walking might produce different walking patterns compared with over-ground 256 walking, although some studies suggested that treadmill walking was very similar 257 to over-ground walking in terms of kinematics and kinetics (Watt et al., 2010). To 258 minimize the adaptation effect between treadmill and over-ground walking, the 259 participants were given sufficient time to customize the walking mode from the 260 treadmill (i.e., walking intervention) to over-ground (i.e., gait experiment) in the 261 present study. Further studies can consider the use of instrumented treadmills (i.e. 262 with embedded force platform) to assess the variability continuously without the 263 need of changing between treadmill and over-ground walking modes.

264 Future studies can apply continuous assessment methods, such as the maximum 265 Floquet multiplier (Bruijn et al., 2013), and establish a cut-off value to identify the 266 instants of possible fatigue, in addition to some feedback questionnaires, such as 267 the Borg perceived exertion scale (Garnacho-Castaño et al., 2018) and fatigue 
268 assessment scale (Marcellis et al., 2015). Alternatively, wearable biofeedback 269 devices can also provide continuous assessment and stimulus and thus enhance 270 sensory input (Ma et al., 2015; Wan et al., 2016). Some investigations are required 271 to investigate if these devices can reduce gait variability and asymmetry, and 272 potentially reduce the risk of falls.

\section{5. Conclusion}

274 After 30 minutes of walking (30-min), the older adults demonstrated a higher inter$275 \operatorname{limb}$ SI, which was predominantly contributed by the ankle joint. Their joint motion 276 was less stable at the knee of the non-dominant side and the ankle of the dominant 277 side, as indicated by the average MAD of the joint angular velocities. Continue 278 walking for an additional 30 minutes $(60-\mathrm{min})$ further deteriorate the stability of the 279 non-dominant limb at the knee joint, as demonstrated by the maximum MAD.

280 Conversely, despite statistical significance not achieved, SI and MAD of the ankle 281 and hip joints deteriorated after 30 minutes of walking and improved after 60 282 minutes of walking, which could be due to an alleged compensatory walking 283 response to maintain balance in gait.

\section{6. Acknowledgement}

285 The work was supported by The Health and Medical Research Fund (HMRF) of 286 Hong Kong, China (11122231) and Beijing Municipal Science and Technology 287 Commission (Z181100009218005). 
290

291

292

293

294

295

296

297

298

299

300

301

302

303

304

305

306

307

308

309

310

311

312

313

314

315

316

317

318

319

320

321

322

323

324

325

326

327

328

329

330

331

332

Ambrose, A.F., Paul, G., Hausdorff, J.M., 2013. Risk factors for falls among older adults: a review of the literature. Maturitas 75, 51-61.

Beauchet, O., Allali, G., Annweiler, C., Bridenbaugh, S., Assal, F., Kressig, R.W., Herrmann, F.R., 2009. Gait variability among healthy adults: low and high strideto-stride variability are both a reflection of gait stability. Gerontology 55, 702-706. Bohm, S., Mademli, L., Mersmann, F., Arampatzis, A., 2015. Predictive and reactive locomotor adaptability in healthy elderly: a systematic review and metaanalysis. Sports Medicine 45, 1759-1777.

Bruijn, S., Meijer, O., Beek, P., Van Dieën, J., 2013. Assessing the stability of human locomotion: a review of current measures. Journal of The Royal Society Interface 10, 20120999.

Chapman, J.P., Chapman, L.J., Allen, J.J., 1987. The measurement of foot preference. Neuropsychologia 25, 579-584.

Chastin, S.F., De Craemer, M., De Cocker, K., Powell, L., Van Cauwenberg, J., Dall, P., Hamer, M., Stamatakis, E., 2019. How does light-intensity physical activity associate with adult cardiometabolic health and mortality? Systematic review with meta-analysis of experimental and observational studies. Br J Sports Med 53, 370376.

Cortes, N., Onate, J., Morrison, S., 2014. Differential effects of fatigue on movement variability. Gait \& Posture 39, 888-893.

Donath, L., van Dieën, J., Faude, O., 2016. Exercise-based fall prevention in the elderly: what about agility? Sports Medicine 46, 143-149.

Duncan, M.J., Kline, C.E., Vandelanotte, C., Sargent, C., Rogers, N.L., Di Milia, L., 2014. Cross-sectional associations between multiple lifestyle behaviors and health-related quality of life in the 10,000 Steps cohort. PloS one 9, e94184.

Elhadi, M.M., Ma, C.Z., Wong, D.W., Wan, A.H., Lee, W.C., 2016. Comprehensive Gait Analysis of Healthy Older Adults Who Have Undergone Long-Distance Walking. Journal of Aging and Physical Activity.

Fallah-Yakhdani, H.R., Abbasi-Bafghi, H., Meijer, O.G., Bruijn, S.M., van den Dikkenberg, N., Benedetti, M.-G., van Dieën, J.H., 2012. Determinants of cocontraction during walking before and after arthroplasty for knee osteoarthritis. Clinical Biomechanics 27, 485-494.

Garnacho-Castaño, M.V., Domínguez, R., González, A.M., Feliu-Ruano, R., Serra-Payá, N., Maté-Muñoz, J.L., 2018. Exercise prescription using the Borg rating of perceived exertion to improve fitness. International journal of sports medicine 39, 115-123.

Greene, B.R., McGrath, D., Walsh, L., Doheny, E.P., McKeown, D., Garattini, C., Cunningham, C., Crosby, L., Caulfield, B., Kenny, R.A., 2012. Quantitative falls risk estimation through multi-sensor assessment of standing balance. Physiological Measurement 33, 2049-2063.

Hamacher, D., Singh, N., Van Dieen, J., Heller, M., Taylor, W., 2011. Kinematic measures for assessing gait stability in elderly individuals: a systematic review. Journal of The Royal Society Interface 8, 1682-1698. 
Hamacher, D., Törpel, A., Hamacher, D., Schega, L., 2016. The effect of physical exhaustion on gait stability in young and older individuals. Gait \& Posture 48, 137139. stride-to-stride fluctuations of human walking. Human Movement Science 26, 555589.

Helbostad, J.L., Sturnieks, D.L., Menant, J., Delbaere, K., Lord, S.R., Pijnappels, M., 2010. Consequences of lower extremity and trunk muscle fatigue on balance and functional tasks in older people: a systematic literature review. BMC geriatrics $10,56$.

Jansen, K., De Groote, F., Duysens, J., Jonkers, I., 2014. How gravity and muscle action control mediolateral center of mass excursion during slow walking: $A$ simulation study. Gait \& Posture 39, 91-97.

Krasovsky, T., Baniña, M.C., Hacmon, R., Feldman, A.G., Lamontagne, A., Levin, M.F., 2012. Stability of gait and interlimb coordination in older adults. Journal of neurophysiology 107, 2560-2569.

Lim, K., Taylor, L., 2005. Factors associated with physical activity among older people-a population-based study. Preventive medicine 40, 33-40.

Ma, C.Z.-H., Wan, A.H.-P., Wong, D.W.-C., Zheng, Y.-P., Lee, W.C.-C., 2015. A vibrotactile and plantar force measurement-based biofeedback system: Paving the way towards wearable balance-improving devices. Sensors 15, 31709-31722.

Maki, B.E., 1997. Gait changes in older adults: predictors of falls or indicators of fear? Journal of the American geriatrics society 45, 313-320.

Marcellis, R., van der Veeke, M., Mesters, I., Drent, M., de Bie, R., de Vries, G., Lenssen, A., 2015. Does physical training reduce fatigue in sarcoidosis? Sarcoidosis vasculitis and diffuse lung disease 32, 53-62.

Paterson, K., Hill, K., Lythgo, N., 2011. Stride dynamics, gait variability and prospective falls risk in active community dwelling older women. Gait \& Posture 33, 251-255.

Paw, M.J.C.A., de Jong, N., Schouten, E.G., Hiddink, G.J., Kok, F.J., 2001. Physical exercise and/or enriched foods for functional improvement in frail, independently living elderly: a randomized controlled trial. Archives of physical medicine and rehabilitation 82, 811-817.

Sergi, G., Sarti, S., Mosele, M., Ruggiero, E., Imoscopi, A., Miotto, F., Bolzetta, F., Inelmen, E.M., Manzato, E., Coin, A., 2011. Changes in healthy elderly women's physical performance: a 3-year follow-up. Experimental gerontology 46, 929-933. Smith, J.W., Christensen, J.C., Marcus, R.L., LaStayo, P.C., 2014. Muscle force and movement variability before and after total knee arthroplasty: A review. World J Orthop 5, 69-79.

Tan, M.P., Kamaruzzaman, S.B., Zakaria, M.I., Chin, A.V., Poi, P.J.H., 2015. Tenyear mortality in older patients attending the emergency department after a fall. Geriatrics \& gerontology international.

Tudor-Locke, C., Bassett Jr, D.R., 2004. How many steps/day are enough? Sports Medicine 34, 1-8.

Van Kan, G.A., 2009. Epidemiology and consequences of sarcopenia. JNHA-The Journal of Nutrition, Health and Aging 13, 708-712. 
379 Wan, A.H., Wong, D.W., Ma, C.Z., Zhang, M., Lee, W.C., 2016. Wearable 380 vibrotactile biofeedback device allowing identification of different floor conditions 381 for lower-limb amputees. Archives of physical medicine and rehabilitation.

382 Watt, J.R., Franz, J.R., Jackson, K., Dicharry, J., Riley, P.O., Kerrigan, D.C., 2010. 383 A three-dimensional kinematic and kinetic comparison of overground and treadmill 384 walking in healthy elderly subjects. Clinical Biomechanics 25, 444-449.

385 Winter, D.A., 1991. Biomechanics and motor control of human gait: normal, elderly 386 and pathological.

387 Wong, D.W.-C., Lam, W.K., Yeung, L., Lee, W.C., 2015. Does long-distance 388 walking improve or deteriorate walking stability of transtibial amputees? Clinical 389 Biomechanics 30, 867-873.

390 Yeung, L., Leung, A.K., Zhang, M., Lee, W.C., 2012. Long-distance walking effects 391 on trans-tibial amputees compensatory gait patterns and implications on prosthetic 392 designs and training. Gait \& Posture 35, 328-333.

393

394

395 


\section{$396 \quad$ Figures Legends}

397 Figure 1. The SI of the ankle, knee, and hip angular velocities under baseline, 30$398 \min (30 \mathrm{~m})$ and $60-\min (60 \mathrm{~m})$. Bracket denotes statistically significance in the post 399 hoc analysis with Bonferroni correction at $p<0.017$.

400 Figure 2. Average MAD of the ankle, knee, and hip joints between the non401 dominant and dominant limbs under baseline, 30-min (30m) and 60-min (60m). 402 Significance levels and Chi-square refer to Friedman test comparing average MAD 403 among time points. o denotes outliers; * denotes outliers of extreme values; 404 bracket denotes statistically significance in the post hoc analysis with Wilcoxon 405 signed-rank test and Bonferroni correction at $p<0.017$.

406 Figure 3. Maximum MAD of the ankle, knee, and hip joints between the non407 dominant and dominant limbs under baseline, 30-min (30m) and 60-min (60m). 408 Significance levels and Chi-square refer to Friedman test comparing maximum 409 MAD among time points. o denotes outliers; * denotes outliers of extreme values; 410 bracket denotes statistically significance in the post hoc analysis with Wilcoxon 411 signed-rank test and Bonferroni correction at $p<0.017$. 


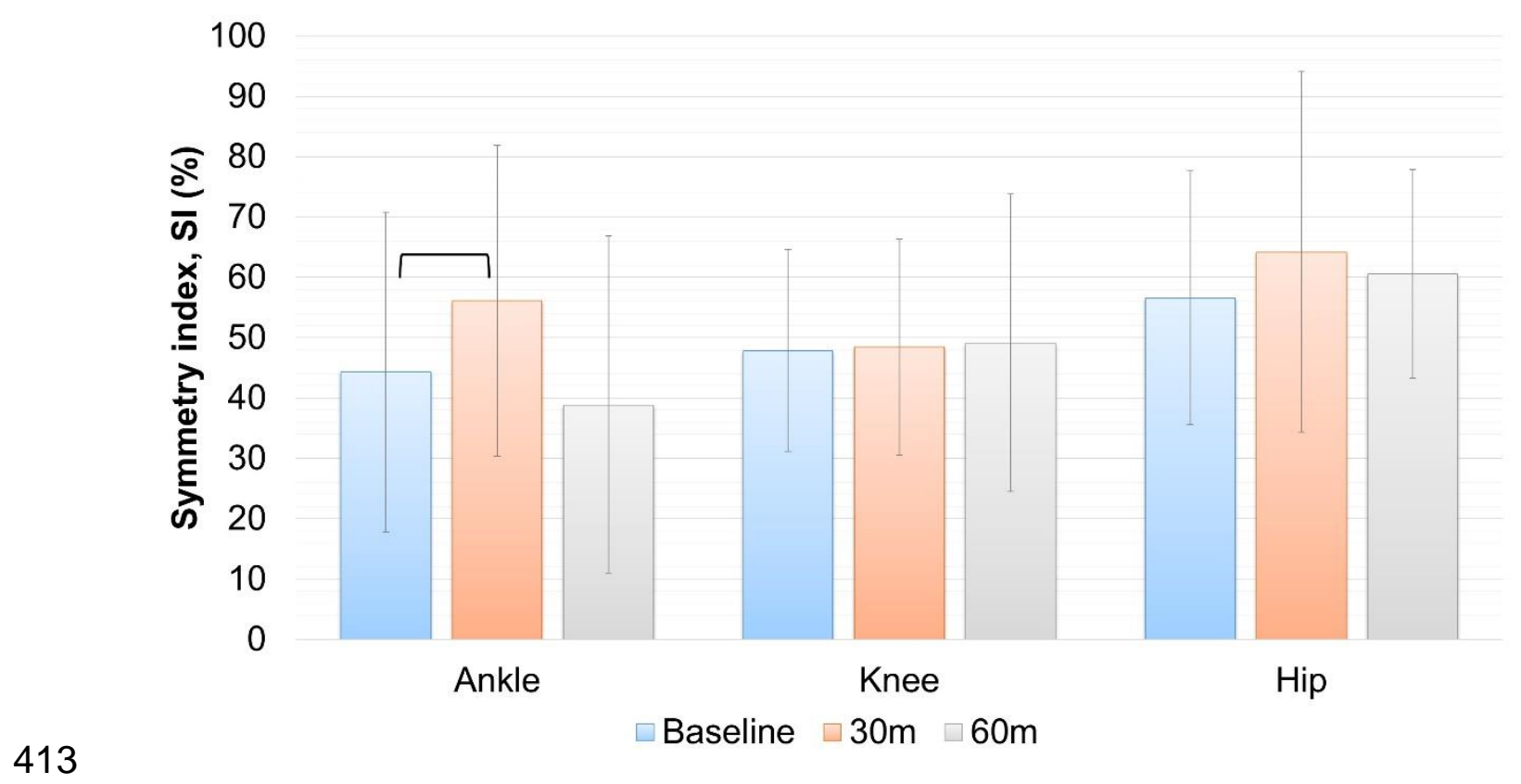

414 Figure 1

415 

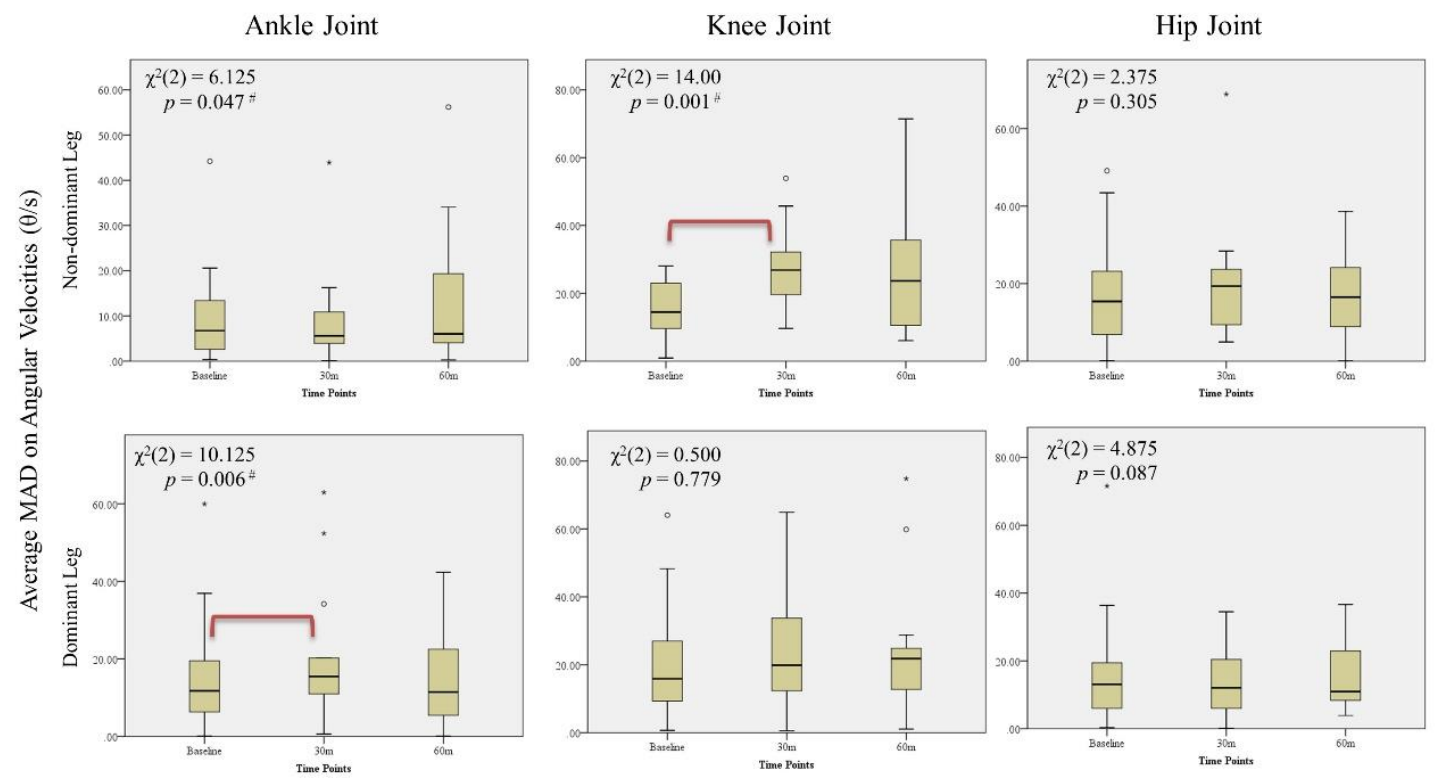

416

$417 \quad$ Figure 2

418 

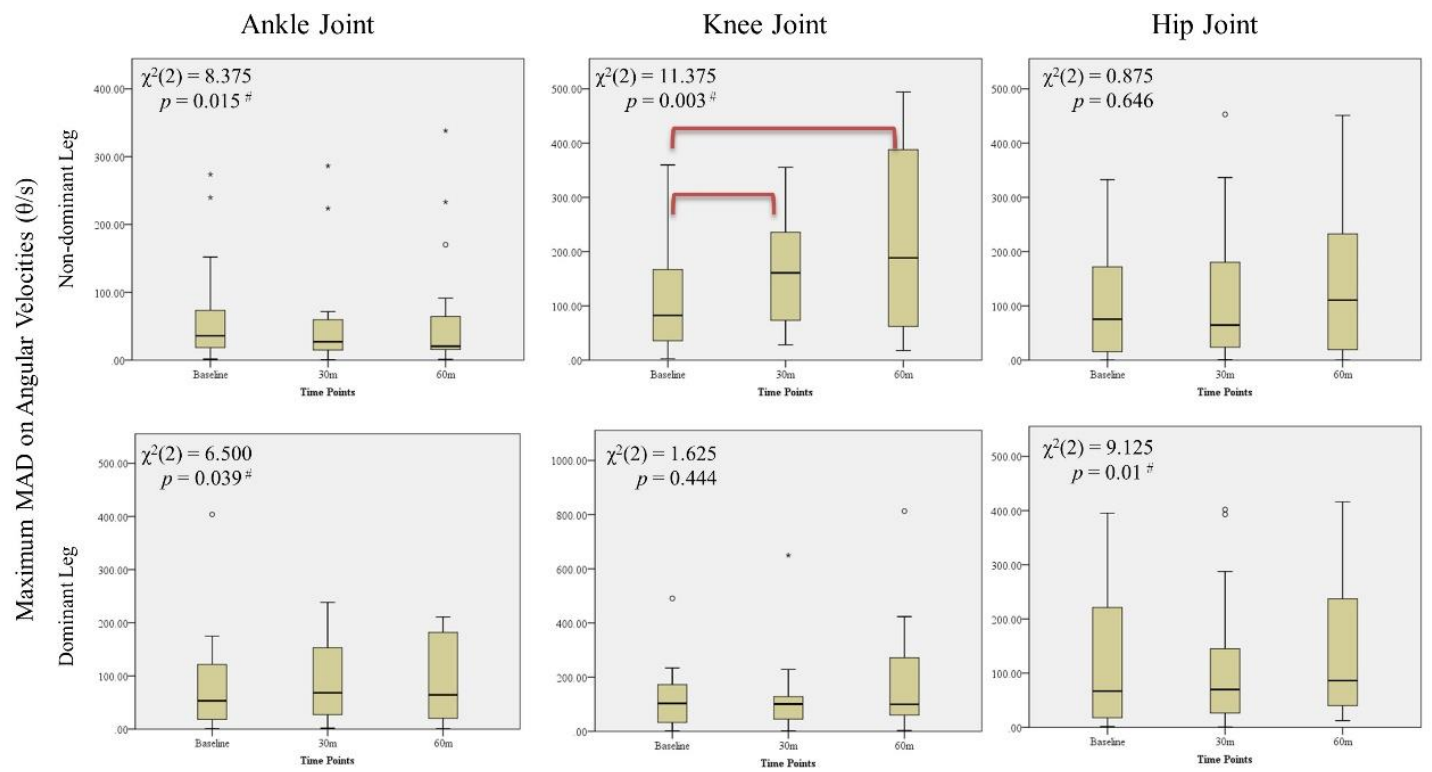

419

$420 \quad$ Figure 3

421 
422 Table 1 Effect of walking time (baseline, 30-min and 60-min) on the spatio423 temporal parameters, including walking speed, step length and stance time of the 424 dominant and non-dominant limbs.

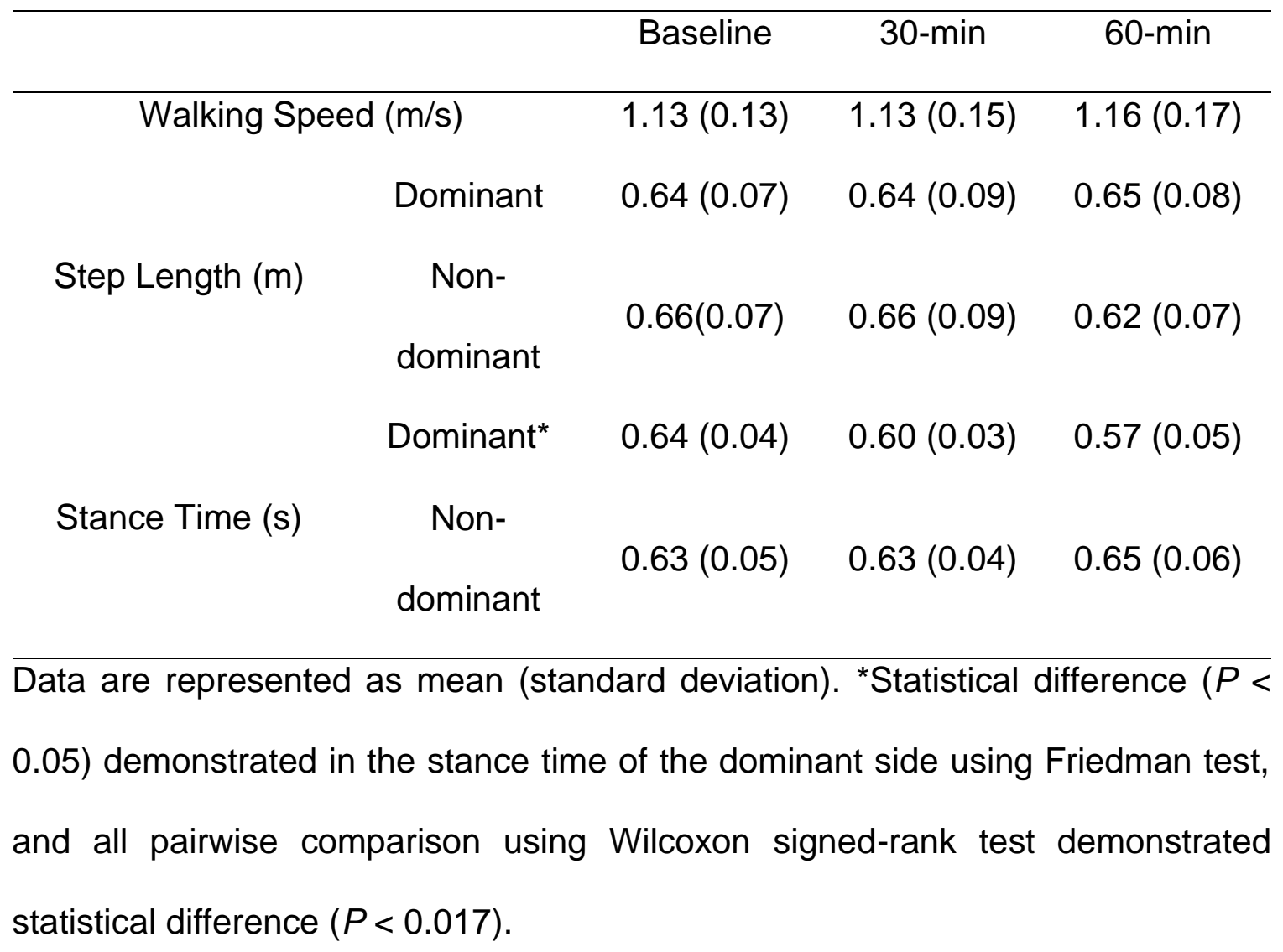

429

430 
431 Table 2 Effect of walking time (baseline, 30-min and 60-min) on the SI of the 432 angular velocities of the ankle, knee and hip joints.

\begin{tabular}{lcccc}
\hline Dependent Variable & $d f$ & $F$ & Partial $\eta^{2}$ & $P$ \\
\hline Combined SI & 6 & 5.47 & 0.77 & $0.010^{*}$ \\
SI of Ankle & 1.31 & 6.58 & 0.31 & $0.013^{\dagger}$ \\
SI of Knee & 2 & 0.06 & 0.00 & 0.940 \\
SI of Hip & 1.34 & 0.68 & 0.04 & 0.462
\end{tabular}

433 Significance level of the combined effect refers to one-way MANOVA repeated 434 measures among the time conditions, and that of the Sls refer to the follow-up 435 univariate tests. ${ }^{*} P<0.05 ;{ }^{\dagger} P<0.017$.

436

437 